\title{
Identification of nanoindentation-induced phase changes in silicon by in situ electrical characterization
}

\author{
S. Ruffell, ${ }^{\text {a) }}$ J. E. Bradby, N. Fujisawa, and J. S. Williams \\ Department of Electronic Materials Engineering, Research School of Physical Sciences and Engineering, \\ Australian National University, Canberra 0200, Australia
}

(Received 21 December 2006; accepted 6 March 2007; published online 30 April 2007)

\begin{abstract}
In situ electrical measurements during nanoindentation of Czochralski grown $p$-type crystalline silicon (100) have been performed using a conducting diamond Berkovich indenter tip. Through-tip current monitoring with a sensitivity of $\sim 10 \mathrm{pA}$ and extraction of current-voltage curves at various points on the complete load-unload cycle have been used to track the phase transformations of silicon during the loading and unloading cycle. Postindent current-voltage curves prove to be extremely sensitive to phase changes during indentation, as well as to the final phase composition within the indented volume. For example, differences in the final structure are detected by current-voltage measurements even in an unloading regime in which only amorphous silicon is expected to form. The electrical measurements are interpreted with the aid of previously reported transmission electron microscopy and Raman microspectroscopy measurements. (C) 2007 American Institute of Physics. [DOI: 10.1063/1.2724803]
\end{abstract}

\section{INTRODUCTION}

Silicon is a technologically important material and is the workhorse of the semiconductor industry. There has been substantial interest in its mechanical properties, most particularly the phase transformations that occur under high pressure loading and on subsequent pressure release. Indentation experiments have revealed that $\mathrm{Si}-\mathrm{I}$ transforms to a metallic Si-II phase on loading when a pressure of $\sim 11 \mathrm{GPa}$ is attained under the indenter tip. Further phase transformations are possible on unloading. For fast unloading the metallic phase transforms to $a$-Si but on slow unloading transforms to a mixture of high pressure polycrystalline phases ( $\mathrm{Si}-\mathrm{III}$ and $\mathrm{Si}-\mathrm{XII}) .{ }^{1-8}$ This pressure-induced crystallization is of great interest and workers have investigated the transformation pathways, ${ }^{4-6,9-13}$ but the exact mechanisms behind the phenomenon are not understood. In situ characterization techniques are required to complement ex situ measurements such as Raman microspectroscopy and cross-sectional transmission electron microscopy, in order to study these phase transformations especially as nanoindentation dimensions are reduced and ex situ measurements become prohibitively difficult. Electrical characterization is proving to be a suitable in situ technique, having high sensitivity to structural changes in the indented material. ${ }^{10,11,14-16}$

Gridneva et al. ${ }^{17}$ made in situ electrical measurements during indentation in silicon. Later other authors ${ }^{9,11}$ investigated the phase transformation from $\mathrm{Si}-\mathrm{I}$ to metallic $\mathrm{Si}-\mathrm{II}$ by indenting in and/or between metal Schottky contacts evaporated on the silicon surface. Increases in current between the two metal contacts, as well as the switching of the $I-V$ characteristics from rectifying to Ohmic behavior, were observed upon transformation from $\mathrm{Si}$-I to $\mathrm{Si}$-II. None of these studies directly correlated the electrical measurements with the load/

\footnotetext{
${ }^{a)}$ Author to whom correspondence should be addressed; FAX: +61 (0) 2-6125-0511; electronic mail: simon.ruffell@anu.edu.au
}

unload curves. Bradby et al. ${ }^{10}$ developed an in situ electrical characterization method which is sensitive to the $\mathrm{Si}$-I to $\mathrm{Si}$-II phase transformation by utilizing the Schottky to Ohmic transformation for reverse-biased evaporated $\mathrm{Al}$ contacts on $\mathrm{Si}$ during indentation loading. A sudden increase in current flow through the contact occurs with the Si-I to Si-II phase transformation. The technique has proven to be effective in monitoring this phase transformation, where the conductivity of the silicon increases by many orders of magnitude. It lacks the sensitivity, however, required to detect the presence of high pressure crystalline phases formed during unloading because the technique relies on the ratio of the resistance of the indented material to the resistance of the rest of the silicon substrate to be of similar magnitude to the ratio of the indented area to the $\mathrm{Al}$ contact area (i.e., $>10^{6}$ ). Mann et al. ${ }^{14,15}$ used a conducting indenter tip (vanadium carbide) to monitor contact resistance during indentation in silicon, as well as monitoring the $I-V$ contact characteristics during the load/unload cycle. This study provided some electrical data and possible insights into the phase transformation mechanisms, but postindentation measurements were not made.

In the present study, we have used a doped diamond nanoindenter tip to measure current flow during nanoindentation and to monitor both the $I-V$ characteristics of the tip/ sample system and the mechanical response of the sample at all stages during indentation. This study presents and interprets electrical behavior during indentation with the aid of previous postindentation cross-sectional transmission electron microscopy (XTEM) and Raman data. Behavior is identified that cannot be obtained from postindentation measurements. The technique opens up prospects to study phase transformations in silicon at the nanometer scale where correlation of indentation data with XTEM and Raman data is prohibitively difficult. For example, a recent study with microscale indentations showed that volumes of high pressure crystalline phases can form within the phase transformed 
zone even during rapid unloading where pop-outs (associated with the formation of high pressure phases) do not occur. These small volumes were only observable by XTEM and undetected by Raman microspectroscopy. ${ }^{18,19}$ Although, for the nanoscale indentations in this study, XTEM cannot be performed, we show that electrical measurements are sensitive to formation of small volumes of high pressure crystalline phases (in the regime where no pop-out events occur) at the nanoscale. Identification of phase transformations via this in situ technique also eliminates ambiguities associated with possible phase modifications that can occur during postindentation sample preparation required for ex situ techniques such as XTEM (e.g., changes in stress conditions around the indent and electron/ion beam induced annealing). Finally, initial measurements indicate that the electrical signatures can be correlated with the residual depth of the indent measured from the load/unload curves (determined by the composition of the phase transformed volume).

\section{EXPERIMENT}

All indentation was performed at room temperature and pressure using a Hysitron Triboindenter fitted with a conducting diamond Berkovich tip (boron doped to a nominal resistivity of $3.3 \Omega \mathrm{cm}$ ). The experimental setup has been described in more detail previously. ${ }^{16}$ All indentation cycles presented here have a maximum load of $10 \mathrm{mN}$ resulting in a penetration depth of $\sim 200 \mathrm{~nm}$.

All samples were cleaved from a $\operatorname{Si}(100) p$-type wafer, $500 \mu \mathrm{m}$ thick with a $7 \mu \mathrm{m} p$-type epilayer. The substrate and epilayer were boron doped to resistivities of 0.006 and $5 \Omega \mathrm{cm}$, respectively. Ohmic contacts were created by thermal evaporation of $\sim 100 \mathrm{~nm}$ of aluminium on to the highly doped back side of the samples. Samples were electrically connected to the indenter stage via conducting silver paste.

Electrical measurements were taken in two modes. The first was to measure the current flowing through the indenter tip and sample during the indentation cycle with a constant voltage applied across the circuit. Features on the current versus time curves are correlated with those on the load/ unload curves. Here, the current flows through a series of resistances including those associated with phase transformed silicon and the interfaces between these volumes. ${ }^{16} \mathrm{~A}$ further effect is the variation during indentation of the tip/ sample contact area, which adds a spreading resistance component to be considered in the analysis of the $I$ vs $t$ data. This surface contact area and associated spreading resistance component change with loading and this behavior, including interpretation of data, is discussed in detail elsewhere. ${ }^{16}$ The second mode involves sweeping the applied voltage (from -8 to $+8 \mathrm{~V})$ at different positions during the load-unload cycle to extract $I-V$ curves. The sweeps were each performed in $\sim 0.1 \mathrm{~s}$. The sweep time does not affect the $I-V$ characteristics. Monitoring of the electrical characteristics during indentation cycles under a range of applied voltages yields no changes in behavior, thus verifying that resistive heating during these measurements is also negligible. These $I-V$ curves can provide information on the electrical barriers between interfaces in the circuit, e.g., the tip/sample, transformed

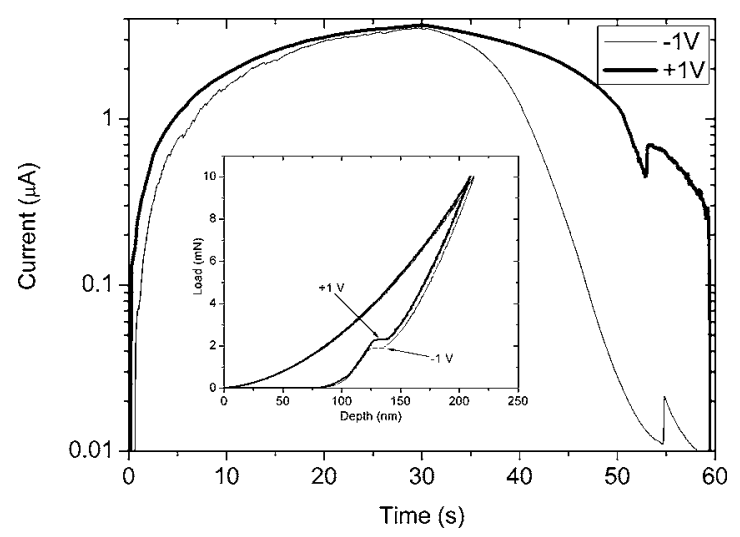

FIG. 1. Current vs time measured through the $c$-Si sample and indenter tip during indentation to a maximum load of $10 \mathrm{mN}$. Loading was performed in $30 \mathrm{~s}$ and unloading in $30 \mathrm{~s}$ at a constant rate $(0.33 \mathrm{mN} / \mathrm{s})$. The curves are shown for an indentation performed with $-1 \mathrm{~V}$ applied to the sample and for an indentation during which $+1 \mathrm{~V}$ was applied. The increase in current observable for both cases towards the end of the cycle corresponds to a pop-out event during unloading. The load/unload curves corresponding to each $I$ vs $t$ curve are shown in the inset.

silicon/silicon matrix interfaces. Pre- and postindentation $I-V$ curves were extracted by holding the load at $0.1 \mathrm{mN}$. This load was chosen so that minimal silicon material deformation occurs (a load/unload cycle to $0.1 \mathrm{mN}$ is completely elastic). Test measurements were made at loads ranging from 0.05 to $0.2 \mathrm{mN}$ with little observable differences in the extracted $I-V$ characteristics indicating that the pressures induced are insufficient to cause measurable changes in the electrical properties of the silicon under test. A small load is essential since a finite contact area is required to make the electrical measurements. Samples were etched in $48 \% \mathrm{HF}: \mathrm{H}_{2} \mathrm{O}$ (1:10) immediately before measurements to remove the native oxide. It has been shown previously that the surface oxide layer (1.5-2 nm thick) affects the electrical measurements made at low loads. ${ }^{16}$

\section{RESULTS}

\section{In situ measurements}

Data shown in Figs. 1-3 were taken during an indentation cycle with a maximum load of $10 \mathrm{mN}$ and a loading and unloading time of $30 \mathrm{~s}$ each. Figure 1 shows current versus time plots taken during indentation in $c$-Si with +1 and $-1 \mathrm{~V}$ applied to the sample. For both voltages, an increase in current is observed almost immediately and the current has a comparable magnitude during loading for both biasing conditions. The current continues to increase during loading up to the maximum load of $10 \mathrm{mN}$ attained after $30 \mathrm{~s}$. The current decreases at a greater rate on unloading for the $-1 \mathrm{~V}$ case than for the $+1 \mathrm{~V}$ curve. Corresponding load/unload curves are shown in the inset. The increases in current at $\sim 52$ and $\sim 55 \mathrm{~s}$, for the +1 and $-1 \mathrm{~V}$ curves, respectively, coincide with the pop-outs on the corresponding load/unload curves (shown in the inset). There is no appreciable difference in the mechanical response under each of the biasing conditions, except for the slight difference in the load at pop-out. 
(a)

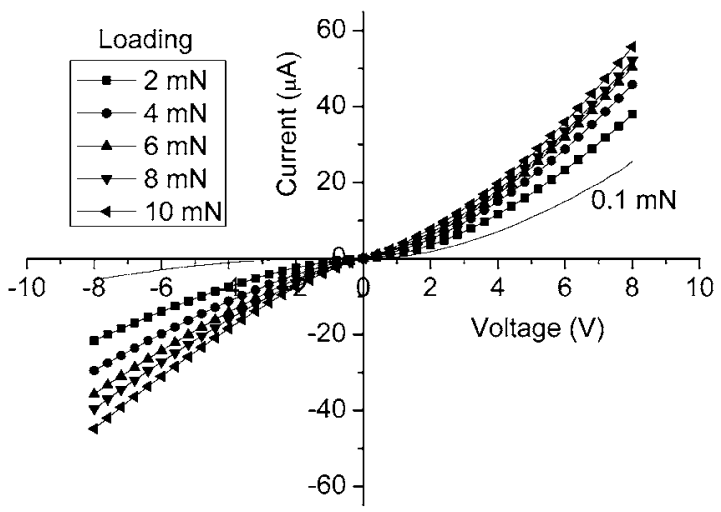

(b)

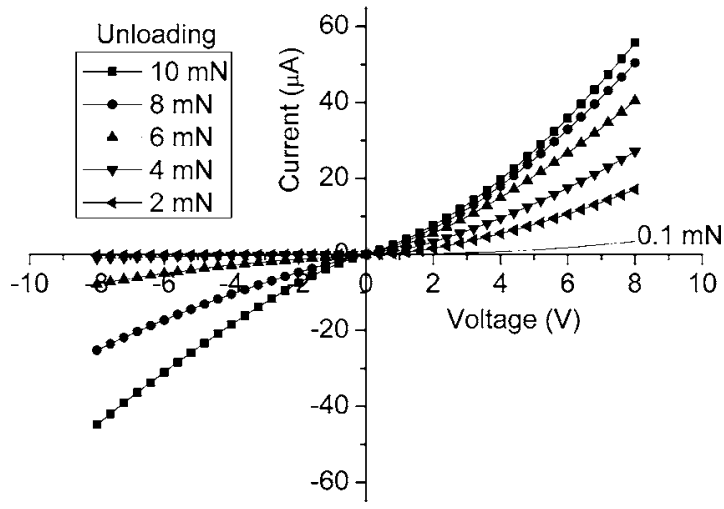

FIG. 2. Current-voltage curves extracted at various points on loading and unloading during an indentation cycle to a maximum load of $10 \mathrm{mN}$ at a constant loading/unloading rate of $0.33 \mathrm{mN} / \mathrm{s}$. (a) shows the $I-V$ curves extracted at $2 \mathrm{mN}$ increments during loading including the preindentation $I-V$ curve extracted at a load of $0.1 \mathrm{mN}$ (solid curve). (b) shows $I-V$ curves extracted at the same loads during unloading and the postindentation $I-V$ curve at $0.1 \mathrm{mN}$ (solid curve).

Figure 2 shows $I-V$ characteristics taken at various positions on the load/unload cycle for an indentation in which no pop-out occurred on unloading. The contact between the indenter tip and silicon is initially rectifying with a negative voltage (reverse bias), as shown by the $I-V$ curve extracted at a load of $0.1 \mathrm{mN}$. The contact becomes more Ohmic with loading [Fig. 2(a)]. On unloading [Fig. 2(b)], the contact becomes rectifying rapidly, i.e., even after unloading to $8 \mathrm{mN}$. At any given voltage, the current flow on unloading is lower at all loads than during loading. Figure 3 shows the ratio of reverse-biased current to forward-biased current (measured at $\pm 5 \mathrm{~V}$ ) during the load/unload cycle from $I-V$ data taken in Fig. 2. These $I-V$ data are in agreement with the observed $I$ vs $t$ data between forward and reverse biasing (Fig. 1), where reverse biasing results in low currents on unloading.

\section{Pre- and Postindentation}

Figure 4 shows the pre- and postindentation $I-V$ curves and the effect that the unloading rate has on the postindentation electrical properties. Loading to $10 \mathrm{mN}$ was performed in $5 \mathrm{~s}$, with the unloading time ranging from 1 to $60 \mathrm{~s}$ ( $10-0.17 \mathrm{mN} / \mathrm{s}$ unload rate). Although pop-outs may occur at the unloading rates studied here, measurements were pur-

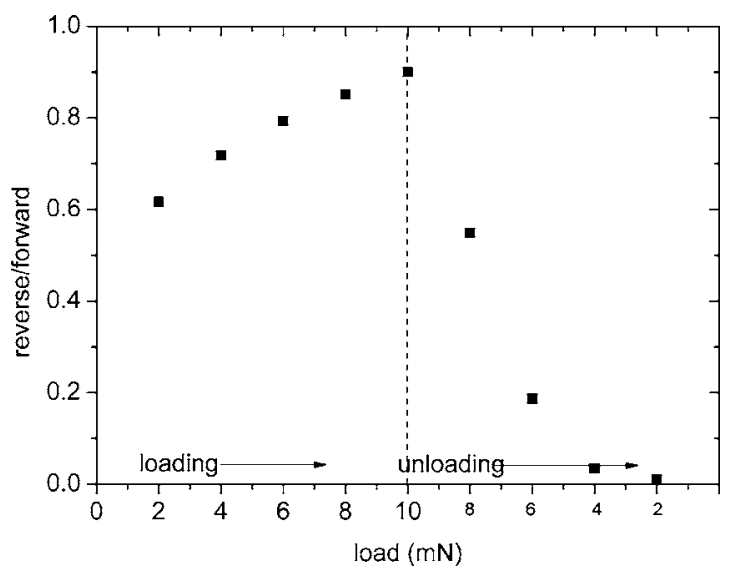

FIG. 3. Ratio of reverse-biased to forward-biased current (measured at $\pm 5 \mathrm{~V}$ ) for each $I-V$ curve extracted during loading and unloading of an indentation cycle (loading to $10 \mathrm{mN}$ in $30 \mathrm{~s}$ with unloading in $30 \mathrm{~s}$ ). On loading the $I-V$ characteristics become more Ohmic and rapidly become rectifying on unloading.

posely made only on indentations where pop-outs did not occur. It has previously been shown that the unloading rate determines the end structure. ${ }^{1-8}$ Slow unloading promotes the "pop-out" event, which is expected to be associated with the formation of substantial volumes of Si-III and Si-XII. ${ }^{1-8}$ Rapid unloading, where no pop-outs occur, is expected to result in the formation of $a$-Si only; ${ }^{1-8}$ however, recent work reported XTEM images showing small volumes of high pressure phases $(<5 \%$ of the phase transformed zone) during certain rapid unloading conditions in which no pop-out occurred. ${ }^{18,19}$ In addition, in another study ${ }^{19}$ in which Raman microspectroscopy measurements were made on arrays of indents in silicon created under the same conditions (from these measurements $\mathrm{Si}$-III/XII are detected in some cases or not detected in others), for unloading rates greater than $1 \mathrm{mN} / \mathrm{s}$ (unload time of $10 \mathrm{~s}$ ) no high pressure phases were detected (i.e., if volumes of high pressure phases were present the amount was below the detection threshold for Raman). The electrical measurements here (Fig. 4) also show that high pressure phases must be forming in this unloading regime as no difference in electrical signature would be observed for indents composed solely of $a$-Si.

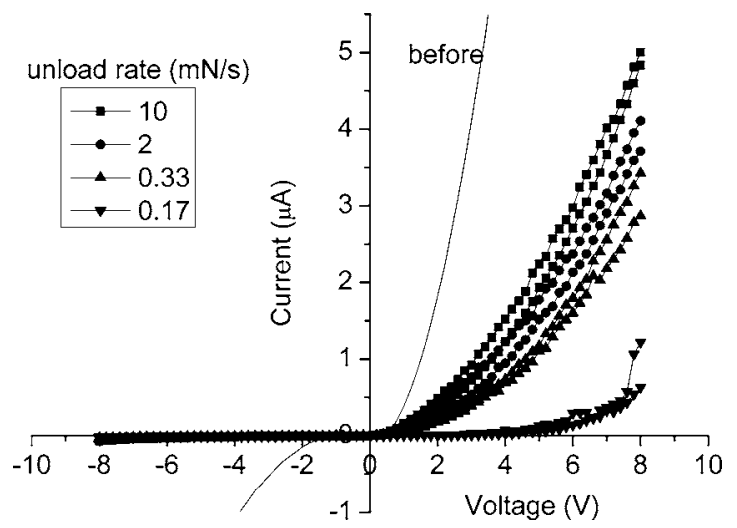

FIG. 4. Pre- and postindentation $I-V$ curves, extracted by holding at a load $0.1 \mathrm{mN}$, as a function of unloading rate. All preindent curves are the same but postindentation characteristics vary with unloading conditions. Greater forward-biased current flows as the unloading rate increases. 
(a)

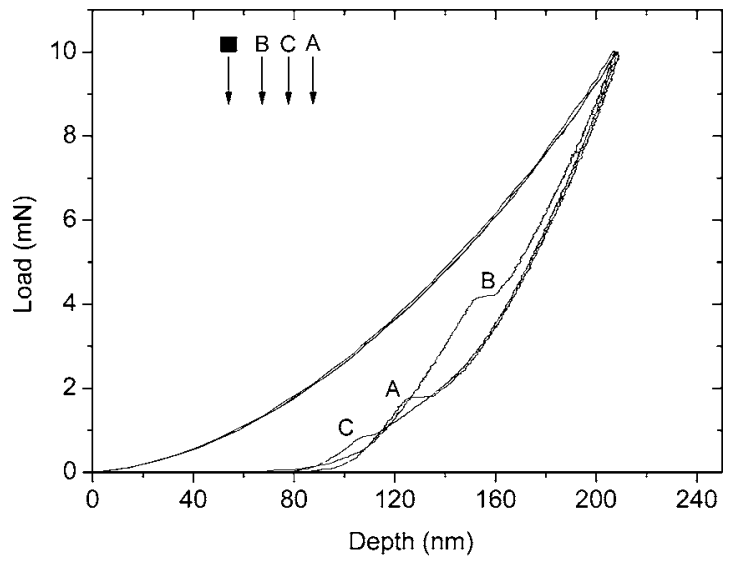

(b)

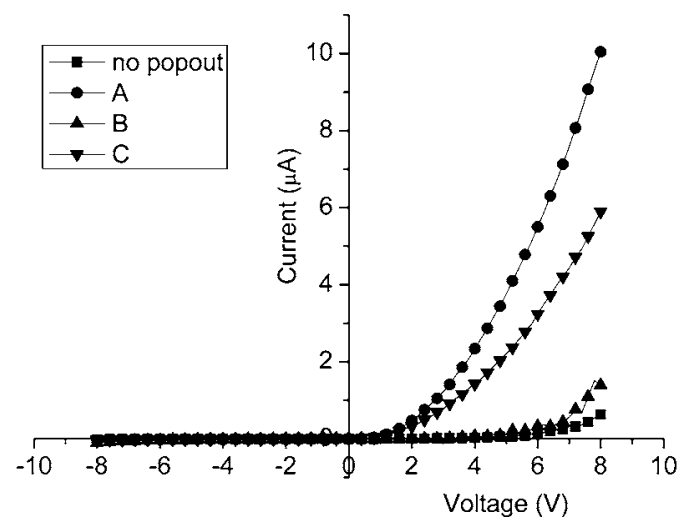

FIG. 5. Load/unload curves and postindentation electrical characteristics for three indentations where pop-out events occurred on unloading (unloading rate of $0.17 \mathrm{mN} / \mathrm{s}$ ). (a) Load/unload curves are shown with the residual indents marked with arrows [also shown is the residual depth for the indentation where no pop-out occurred-shown as $\boldsymbol{\square}$ in (b)]. The curves are labeled A, B, and C. Corresponding postindentation $I-V$ characteristics are shown in (b). The forward-biased current increases with residual depth. For curve B (smallest residual depth) the $I-V$ characteristics are similar to that where a pop-out did not occur.

Close inspection of the load/unload curves obtained from this experiment reveals small differences in the mechanical response. In particular, the final depth (residual depth of indent) varies with the unload rate. Amorphous silicon is $24 \%$ less dense than $\mathrm{Si}-\mathrm{II}$ and $\mathrm{Si}-\mathrm{III}$ is $14 \%$ less dense. ${ }^{10}$ The difference in densities results in the residual depth of the indent being partly determined by the composition of the phase transformed zone below the surface, e.g., a phase transformed zone of $a$-Si will result in a smaller residual depth than that composed of Si-III, for similar maximum load conditions. It is expected, therefore, that the variations in electrical signature can be correlated with the residual depth (see below).

Figure 5 shows load/unload curves [Fig. 5(a)] and postindentation $I-V$ characteristics [Fig. 5(b)] for three indentations for which pop-out events occurred during unloading. The residual indent depths are marked on the load/ unload curves in Fig. 5(a). For pop-outs A and C, the residual depths are greater than any of those for which pop-outs did not occur (79 and $89 \mathrm{~nm}$ compared to a maximum residual depth of $70 \mathrm{~nm}$ for the non-pop-out indentations). As with

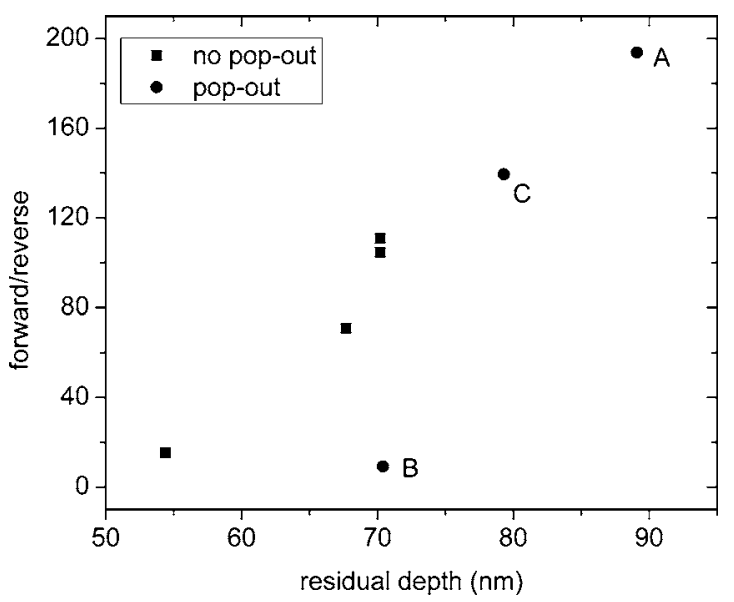

FIG. 6. Forward to reverse current measured after indentation (by holding at $0.1 \mathrm{mN}$ ) vs residual indent depth. The ratio increases monotonically with increasing residual depth. The high pressure crystalline phases ( $\mathrm{Si}$-III and $\mathrm{Si}-\mathrm{XII})$ are more conductive than $a$-Si so indents containing more of Si-III/ XII will result in greater forward-biased current. These high pressure phases are more dense than $a$-Si so a greater residual depth indicates a larger volume of $\mathrm{Si}-\mathrm{III} / \mathrm{XII}$.

the indentations in which no pop-out occurs, the forwardbiased current increases with increasing residual depth [Fig. $5(b)]$. For curve B where the pop-out occurs earlier on unloading, the residual depth is comparable to those with no pop-outs. Correspondingly, the $I-V$ characteristic is similar to those extracted from indentations with no pop-outs.

Care must be taken in interpretation of the postindentation $I-V$ characteristics, as differences in residual depth will result in differences in the topography of the indent and, therefore, differences in the tip/sample contact area. It is unlikely that the variation in contact area here is large enough to account for the differences in the electrical signature observed, as we indicate. One way to account for this, however, is to extract the rectification ratio (ratio of forward to reverse-biased current) as this is independent of magnitude of current (determined by the contact area) and will be determined by the electrical properties of the material beneath the indenter tip.

Figure 6 shows the rectification ratio (forward current/ reverse current) from the $I-V$ data extracted from postindentation at different unloading rates where no pop-outs occurred (Fig. 4), as well as those in which pop-outs occurred (Fig. 5), as a function of residual depth. Although only a small amount of data was taken to extract these results, a trend is observed. The rectification increases (i.e., greater forward-biased current) with increasing residual depth. In addition the data fall into two regimes, pop-outs and non-popouts. For indentations where pop-outs occur, the exact microstructure and depth distribution will differ to those where no pop-outs occur, thus producing different absolute $I-V$ characteristics.

\section{DISCUSSION}

\section{In situ measurements}

Before a discussion of the interpretation of the electrical measurements, it is useful to review the indentation mechan- 
(a) Loading

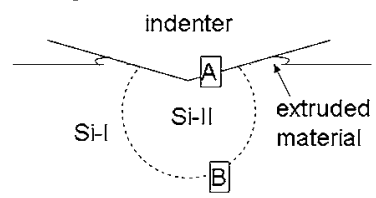

(b) Rapid unloading

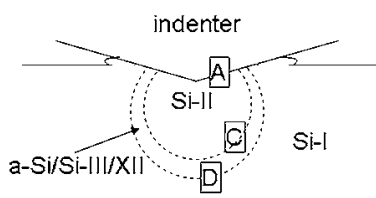

(c) Slow unloading

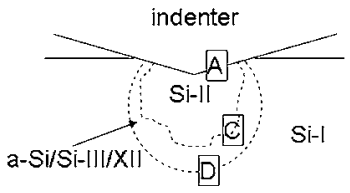

FIG. 7. Schematic showing volumes of phase transformed material and the interfaces determining the measured current during loading and unloading. For loading (a) there are two interfaces. A-between the indenter tip and $\mathrm{Si}-\mathrm{II} ; \mathrm{B}$-between the $\mathrm{Si}-\mathrm{II}$ volume and the surrounding $\mathrm{Si}$ matrix. The unloading case is slightly more complicated [(b) and (c)]. C-an interface between the remaining $\mathrm{Si}-\mathrm{II}$ and the further transformed region around this $\mathrm{D}$ - the further transformed region $(a-\mathrm{Si}$ for fast unloading and $a$-Si/Si-III/Si-XII for slow unloading) and the surrounding Si matrix. Later in unloading interface A will be between the tip and some further transformed material.

ics and the associated phase transformations that occur in silicon under indentation. At a pressure of $\sim 11 \mathrm{GPa}, \mathrm{Si}-\mathrm{I}$ transforms to metallic Si-II. This occurs during the early stages of loading with sharp Berkovich tips, giving rise to a volume of Si-II almost immediately and the volume increases in size with loading. On unloading, the pressure release causes further phase transformations from $\mathrm{Si}$-II to $\mathrm{Si}$ III, Si-XII, and $a$-Si. The final structure is unload-rate dependent, with slow unloading favoring formation of a mixture of Si-III/XII crystalline phases and fast unloading resulting in formation of $a$-Si only. ${ }^{1-4,6-8}$ Therefore, the phase composition and grain size, as well as the nature of interfaces between the various material phases, depend critically on the load/unload cycle. These phases are shown schematically for loading and unloading in Fig. 7. The conductivity of these phases and the nature of the electrical barriers formed at the interfaces between them dominate the electrical characteristics. Thus, in principle electrical measurements allow phase transformations and phase composition to be monitored during the whole indentation cycle.

The electrical measurements provide valuable in situ measurements but also ex situ characterization and provides a means for correlating measurements of these nanosized domains with load/unload curves. Raman micro spectroscopy and XTEM, the traditional ex situ characterization techniques for nanoindents, cannot currently be used for characterization of individual nanosized domains; however, development of sample preparation to achieve XTEM characterization is under way.

\section{Loading}

Evidence for conductivity changes during loading is extracted from the $I-V$ characteristics measured throughout the load/unload cycle. As the load increases, the $I-V$ curves tend to Ohmic behavior from the initial rectifying characteristic. This rectifying characteristic originates from the contact between a highly doped indenter tip (having almost metallic properties ${ }^{16}$ ) and the $p$-type silicon, thus creating a Schottky contact. $^{20}$ At maximum load, a relatively good Ohmic characteristic is measured, indicating that potential barriers between the tip and metallic Si-II, as well as $\mathrm{Si}-\mathrm{II}$ and the surrounding Si-I matrix, are not significant. Once a pressure is reached at which a transformation from $\mathrm{Si}$-I to $\mathrm{Si}$-II has occurred, one would expect Ohmic behavior to dominate unless a significant fraction of the indenter tip is also in contact with untransformed $\mathrm{Si}$ and extruded material. The exact nature of the extruded material will also determine the partial $I-V$ characteristic between it and the indenter tip. It should be noted that the pressure relief that the extruded material experiences occurs rapidly so is expected to be insulating $a$-Si. ${ }^{10,16}$ However, there have been some reports that suggest the material can be polysilicon or high pressure crystalline phases under some conditions. ${ }^{15,21,22}$ The $I-V$ characteristics, therefore, will be made up of a combination of both rectifying (from contact to Si-I and extruded material) and Ohmic characteristics (from contact with $\mathrm{Si}-\mathrm{II}$ ). This appears to be the case during loading in Fig. 3. Furthermore, the fraction of the tip in contact with Si-II will depend on the amount of extruded material and the tip geometry, both of which are difficult to extract. ${ }^{16}$

\section{Unloading}

The results here show that, during unloading, electrical changes are clearly observed and these relate to phase changes and changes in contact area, but interpretation of the electrical measurements and their relation to the composition of the material under the indenter tip are complex. Figures 7(b) and 7(c) show a number of possible interfaces created between the various phases of silicon during unloading.

For a bias of $-1 \mathrm{~V}$, the current drops rapidly on unloading, indicating the formation of a more resistive material (than $\mathrm{Si}$-II) and/or an electrical barrier between a transformed phase and the surrounding material. The changes in electrical signature from the $I-V$ characteristics also occur immediately after unloading begins, suggesting detection of phase changes (to $a$-Si and/or Si-III/XII) initially at the periphery of the phase transformed Si-II volume [interface B in Fig. 7(a)], i.e., where the pressure first drops below that required to stabilize Si-II. During the early stages of unloading, the material in contact with the indenter tip is still predominantly Si-II. Therefore, the contact resistivity at this point (between the tip and sample) is not expected to change significantly from that at maximum load. The resultant rectification that is observed, therefore, must originate from deeper interfaces between either the $\mathrm{Si}-\mathrm{II}$ and whatever phase $(a-\mathrm{Si}$, $\mathrm{Si}-\mathrm{III} / \mathrm{Si}$-XII) is formed around it, or the latter and the untransformed Si-I matrix. 
It is believed that, on unloading, $a$-Si forms initially since the formation of the high pressure phases involves a nucleation process, ${ }^{1,2,22}$ thus inhibiting their formation until a pop-out event occurs later in the unloading cycle. If this is the case here, the initial change in the $I-V$ characteristics on unloading to rectifying behavior is due to the formation of $a$-Si, since one would expect a Schottky-like junction to be formed similar to that of a metal-thin oxide-silicon structure ${ }^{20}$ Here the Si-II is metallic, with a thin $a$-Si layer between it and the surrounding crystalline silicon matrix. Indeed, we have demonstrated elsewhere that, during indentation of a thin $(\sim 350 \mathrm{~nm})$ surface layer of $a-\mathrm{Si}$ in a $c$-Si substrate (where the tip is in direct contact with a volume of phase transformed metallic silicon), a rectifying $I-V$ characteristic is measured. ${ }^{19}$ It is not known, however, what the $I-V$ characteristic would be if $a$-Si was replaced by high pressure phases $(\mathrm{Si}-\mathrm{III} / \mathrm{XII})$ between the metallic $\mathrm{Si}-\mathrm{II}$ and the surrounding $c$-Si substrate.

Mann et al. ${ }^{14,15}$ attributed the electrical behavior, including rectification observed on unloading, to the formation of first Si-XII followed by Si-III and then the increases in current as a result of the formation of metallic $a-\mathrm{Si}$. The data presented here suggest the opposite. At the point of pop-out (Fig. 1), the current increases and it is now known that this is the point at which a significant volume of $\mathrm{Si}$-III/XII is formed. It has been shown elsewhere that residual indents containing high pressure phases are considerably more electrically conductive than those composed of $a$-Si only. ${ }^{19}$ Hence, if the pop-out is the formation of substantial volumes of high pressure phases, it is expected that the current should increase at pop-out. This behavior (current increase at popout) has also been observed for larger scale indentations studied using a different electrical measurement configuration. ${ }^{23}$ This behavior supports our conclusion that $a$-Si is the dominant result of Si-II phase transformations during the initial stages of unloading and pop-out signifies the first significant transformation to Si-III/XII.

The rectification observed on unloading will be dependent on the unloading conditions since different unloading rates produce a different mix and fraction of phases. This raises the possibility of using postindentation electrical characteristics to provide information on end phases produced during unloading. The following is a discussion of initial results obtained by extracting $I-V$ characteristics postindentation.

\section{Pre- and Postindentation}

Before indentation, the dominant resistance determining the $I-V$ characteristics is that of the indenter tip/c-Si contact. ${ }^{16}$ The ratio of reverse to forward-biased current is found to be $\sim 0.2$ in this case. After indentation, there are two interfaces that the current has to flow through-the tip/ transformed Si (i.e., $a-\mathrm{Si}, \mathrm{Si}$-III/XII, or a mixture of both) interface and the interface between the transformed $\mathrm{Si}$ and the surrounding untransformed $c-\mathrm{Si}$. The $I-V$ characteristic becomes more rectifying (ratio of reverse/forward current decreases to $\sim 0.01$ ) than before indentation in this case (see Figs. 3 and 4).
A second effect observable in the postindentation $I-V$ characteristics, namely, that the forward-biased current observed correlates well with the residual indentation depth. Higher currents correspond to larger residual depths. It is quite unexpected that any variation in the final $I-V$ characteristics should be observed between cases where no pop-out occurs since only $a$-Si would be expected to form without pop-outs. However, the variations observed here with unloading (both differences in the residual indentation depth and electrical characteristics) suggest that it is not only $a-\mathrm{Si}$ that is being formed in the indented region, but rather, some significant volumes of high pressure phases within an $a-\mathrm{Si}$ matrix can be created even without a pop-out event. For the indentations here, the increased residual depth is consistent with a larger volume of high pressure phase within the indent. Indeed, a larger volume of high pressure crystalline phases (Si-III and Si-XII are more dense than $a$-Si by about $12 \%$ ) would give a larger residual depth. Thus, when there is no pop-out, small volumes of high pressure phases may form but are surrounded by a large volume of $a$-Si on unloading. The electrical characteristics in this regime, therefore, are dominated by $a$-Si, but the absolute forward-biased current can increase (possibly by hopping conduction) as the volume of more conducting zones of high pressure phase increases. The trend of increasing forward-biased current is consistent with this scenario since it is expected that the high pressure crystalline phases are more conductive than $a$-Si. Indeed, the electrical measurements appear to be particularly sensitive to relatively small variations in residual depth and hence small amounts of high pressure phases. A detailed study is under way to investigate the relationship between the composition and microstructure of the indented zone and the residual depth of the indent. ${ }^{24}$

A final observation relates to similar correlations between electrical characteristics and residual indent depth after pop-out, despite the limited data. The occurrence of a pop-out is thought to be a sudden formation of a large volume of the high pressure phases and the final structure (and presumably electrical behavior) will be dependent on when (during unloading) this occurs and the phases that form during final stages of unloading. In Fig. 5, after pop-out, the forward-biased current increases with the residual depth (as for cases with no pop-out), despite the fact that the pop-outs occur at different and uncorrelated points on the unloading curve. Thus, despite when the pop-out occurs, the electrical characteristics follow the trend of residual indent depth, suggesting that the volume of high pressure phase in $a$-Si is more important than the precise microstructure in determining the forward-biased currents.

\section{CONCLUSION}

In situ electrical measurements have been made during nanoindentation of $c$-Si using a conducting diamond Berkovich tip. Through-tip current monitoring and extraction of $I-V$ characteristics during the loading cycle have been used to probe pressure-induced phase transformations throughout the complete indentation cycle at the nanometer scale. Experience from previous phase transformation studies conducted 
at the microscale that used XTEM and Raman spectroscopy has been used to assist interpretation of electrical measurements extracted from nanoindentations in the current study. We have shown that this extremely sensitive probe provides a means to track the phase transformations during loading/ unloading and to help us understand the effect of unloading conditions on the final phase, without using ex situ techniques such as XTEM and Raman microspectroscopy, both of which are extremely difficult to perform on nanometer dimensions such as in the present study.

Our results have shown the following: First, forwardbiased current increases with the depth of the residual indent. Second, the $I-V$ characteristics and forward-biased current are extremely sensitive to the volume of high pressure phases within the indentation zone. Third, significant volumes of high pressure phases can be present within the $a$-Si matrix of a residual indent without pop-out and this behavior can be sensitively monitored by electrical measurements. Fourth, in the regime where pop-outs occur the $I-V$ characteristics correlate with the residual indent depth, but they are dependent on the microstructure and depth distribution of the high pressure phases compared to the characteristics extracted when no pop-outs occur where they are dependent on the volume of the phases within the $a$-Si matrix only. Finally, in situ electrical characterization is a powerful means of tracking phase changes during nanoindentation.

\section{ACKNOWLEDGMENTS}

This work was funded by the Australian Research Council and WRiota Pty Ltd. The authors also acknowledge Hysitron Inc. with whom they are developing the in situ electrical measurement system.
${ }^{1}$ V. Domnich, Y. Gogotsi, and S. Dub, Appl. Phys. Lett. 76, 2214 (2000).

${ }^{2}$ J. E. Bradby, J. S. Williams, J. Wong-Leung, M. V. Swain, and P. Munroe, J. Mater. Res. 16, 1500 (2001).

${ }^{3}$ J. E. Bradby, J. S. Williams, J. Wong-Leung, M. V. Swain, and P. Munroe, Appl. Phys. Lett. 77, 3749 (2000).

${ }^{4}$ A. Kailer, Y. G. Gogotsi, and K. G. Nickel, J. Appl. Phys. 81, 3057 (1997).

${ }^{5}$ G. M. Pharr, W. C. Oliver, and D. S. Harding, J. Mater. Res. 6, 1129 (1991).

${ }^{6}$ J. S. Williams, Y. Chen, J. Wong-Leung, A. Kerr, and M. V. Swain, J. Mater. Res. 14, 2338 (1999).

${ }^{7}$ T. Juliano, Y. Gogotsi, and V. Domnich, J. Mater. Res. 18, 1192 (2003).

${ }^{8}$ T. Juliano, V. Domnich, and Y. Gogotsi, J. Mater. Res. 19, 3099 (2004).

${ }^{9}$ D. R. Clarke, M. C. Kroll, P. D. Kirchner, R. F. Cook, and B. J. Hockey, Phys. Rev. Lett. 60, 2156 (1988).

${ }^{10}$ J. E. Bradby, J. S. Williams, and M. V. Swain, Phys. Rev. B 67, 085205 (2003).

${ }^{11}$ G. M. Pharr, W. C. Oliver, R. F. Cook, P. D. Kirchner, M. C. Kroll, T. R. Dinger, and D. R. Clarke, J. Mater. Res. 7, 961 (1992).

${ }^{12}$ E. R. Weppelmann, J. S. Field, and M. V. Swain, J. Mater. Res. 8, 830 (1993).

${ }^{13}$ Y. G. Gogotsi, V. Domnich, S. N. Dub, A. Kailer, and K. G. Nickel, J. Mater. Res. 15, 871 (2000).

${ }^{14}$ A. B. Mann, D. van Heerden, J. B. Pethica, P. Bowes, and T. P. Weihs, Philos. Mag. A 82, 1921 (2002).

${ }^{15}$ A. B. Mann, D. van Heerden, J. B. Pethica, and T. P. Weihs, J. Mater. Res. 15, 1754 (2000).

${ }^{16}$ S. Ruffell, J. E. Bradby, and J. S. Williams, J. Mater. Res. 22, 578 (2007).

${ }^{17}$ I. V. Gridneva, Y. V. Milman, and V. I. Trefilov, Phys. Status Solidi A 14, 117 (1972).

${ }^{18}$ S. Ruffell, J. E. Bradby, and J. S. Williams, Appl. Phys. Lett. 89, 091919 (2006).

${ }^{19}$ S. Ruffell (unpublished).

${ }^{20}$ S. M. Sze, in Physics of Semiconductor Devices (Wiley, New York, 1981).

${ }^{21}$ Y. Gogotsi, T. Miletich, M. Gardner, and M. Rosenberg, Rev. Sci. Instrum. 70, 4612 (1999).

${ }^{22}$ J.-I. Jang, M. J. Lance, S. Wen, T. Y. Tsui, and G. M. Pharr, Acta Mater. 53, 1759 (2005)

${ }^{23}$ B. Haberl, J. E. Bradby, S. Ruffell, J. S. Williams, and P. Munroe, J. Appl. Phys. 100, 013520 (2006).

${ }^{24}$ N. Fujisawa, S. Ruffell, and J. S. Williams (unpublished). 